QUADERNS DE FILOSOFIA VOL. IV NÚM. I (20I7): 47-55

eISSN: 234 I-3042 DOI: I 0.7203/QFIA.4.I.9278

Alfredo Esteve

Universidad Católica de Valencia

\title{
La fundamentación zubiriana de la pedagogía de López Quintás
}

\author{
(The Zubirian Basis of López Quintás's Pedagogy)
}

Recibido: 29/11/16. Aceptado: 20/4/17

Resumen: La enseñanza de la filosofía no es únicamente una transmisión de contenidos; incluso probablemente no sea este el objetivo más importante. Para plantearnos qué es lo más importante en la docencia filosófica deberíamos preguntarnos qué queremos exactamente que aprendan nuestros alumnos. Cuando intentamos responder a esta cuestión se pone de manifiesto la especificidad de la filosofía. La filosofía no es cuestión de contenidos sino de vida; no es cuestión de respuestas, sino de preguntas. En este artículo se pretende mostrar la pedagogía de la admiración de Alfonso López Quintás a la luz de la inteligencia sentiente de Xavier Zubiri.

Abstract: Teaching philosophy is not only about conveying contents; indeed this is probably not the most important goal. In reflecting about what is most important in teaching philosophy we should ask ourselves what we want specifically our students to learn. When we try to answer this question the specificity of philosophy is revealed. Philosophy is not a matter of contents but of life-it is not about answers but about questions. This paper aims to present Alfonso Lopez Quintás's pedagogy of admiration in the light of Xavier Zubiri's sentient intelligence.

Palabras clave: docencia filosofía, Xavier Zubiri, Alfonso López Quintás, capacitación educativa.

Keywords: teaching philosophy, Xavier Zubiri, Alfonso López Quintás, educational training. 


\section{LA ESPECIFICIDAD DE LA DOCENCIA FILOSÓFICA}

D ecientemente leí una $\operatorname{carta}^{1}$ del diario El País en la que una joven de Luelva se quejaba de la situación de desconcierto en que se encontraban no pocos jóvenes de hoy. Manifestaba lo poco que sabían de sí mismos y de la vida, a pesar de los años que pasaban formándose: "Pasamos años en la escuela conociendo mentalidades pero no sabemos explicar lo que nos ocurre, no sabemos escribir, ni escribirnos, no queremos leer nuestra propia vida”. En lugar de ello — continúa - los jóvenes prefieren huir de sí mismos, confiando en un Gobierno que en teoría debería haber velado por ellos en lugar de robar lo más preciado que poseen: 'su palabra, su filosofía'. No podía haber empezado esta reflexión mejor que con las palabras de esta joven, palabras que nos invitan a pensar sobre cuál sea el cometido radical de la filosofía. Porque tal y como ella nos dice, no se trata de 'conocer mentalidades' mientras no queremos leer nuestra propia vida, sino de 'saber de la vida', de 'escribirnos'. Y esto, ¿cómo se enseña?

La filosofía, al igual que cualquier otra disciplina del conocimiento, ha de considerar en su docencia los planteamientos o las herramientas que la pedagogía pueda ofrecer. Lejos de mantener posturas enfrentadas, filosofía y pedagogía pueden establecer un diálogo fecundo y provechoso, siempre que se sea capaz de evitar los dos polos definidos por una mala interpretación del mismo, ambos igualmente rechazables: por un lado, el de ejercer la docencia de la filosofía haciendo caso omiso de las propuestas pedagógicas; por el otro, la disolución de lo específicamente filosófico a causa de las sugerencias establecidas por la disciplina pedagógica (Ruiz López ı988, 287).

Pero si por un lado la filosofía se une así al resto de disciplinas del conocimiento desde el punto de vista pedagógico, por el otro es preciso realizar algunas consideraciones a causa de la especificidad propia de su ejercicio; especificidad propia que se pone de manifiesto cuando nos preguntamos qué pensamos que deben aprender los alumnos de filosofía. Porque la docencia de la filosofía no consiste únicamente en la transmisión de unos contenidos determinados, histórica o sistemáticamente; probablemente, ni siquiera sea este su objetivo principal. Con ello tampoco quiero decir que en el resto de disciplinas lo sea (sería un reduccionismo injusto y falso), pero sí que es cierto que la disciplina filosófica posee una serie de características particulares, como son no únicamente las que tienen que ver con su contenido material — que también-, sino sobre todo con su repercusión vital en las personas implicadas (tanto docentes como alumnos).

${ }^{1}$ Millán Lagares, B., "Filosofía”, [acceso 13.09.2016] http:/lelpais.com/elpais/2016/09/17/opinion/1474132172_164990.html. 
Efectivamente, de lo que se trata no es tanto de aprender contenidos como de integrarlos experiencialmente. ¿Con qué finalidad? Tal y como nos propone Zubiri, más allá de lo académico un espíritu filosófico se articula alrededor de tres ámbitos: las cosas, el mundo y la vida; pero no desde una actitud meramente intelectual, como si quisiéramos elaborar una teoría de las cosas, del mundo o de la vida. No se trata de eso, o no se trata solo de eso; de lo que se trata es de realizar esa elaboración intelectual con una finalidad práctica, experienciada en primera persona: de lo que se trata es de configurar nuestro modo de vida, para lo cual es preciso un saber acerca de las cosas y una orientación en el mundo. El saber no es un fin en sí mismo: no se trata del saber por el saber, sino de su aplicación a nuestro modo de vivir. La filosofía no es el terreno de la posesión intelectual sino el terreno del deseo experiencial, de la inquietud vital. Y el filósofo no se puede mantener al margen en ese proceso: está implicado, totalmente sumergido en eso que podemos denominar experiencia filosófica. Si somos capaces de embarcarnos en ese viaje yendo más allá de los contenidos, nos configuraremos a la vez que vamos tras el conocimiento filosófico. La filosofía es algo en que "teoría y práctica van juntos" (Argullol \& Djermanovic 20i6).

En este viaje, las técnicas pedagógicas son interesantes en la medida en que facilitan esta comunicación de contenidos y actitudes, pero sin perder de vista aquello que le corresponde al alumno: una alternancia entre el esfuerzo intelectual y el reposo reparador, entre la compañía y la soledad, entre el diálogo instructivo y la reflexión personal... Porque si bien no hay que descuidar las prácticas pedagógicas, tampoco caer en el error de atender más a estas que a la propia 'exigencia del pensar' intrínseca al ejercicio de la filosofía. No vayamos a caer en el error de don Avito Carrascal quien pensaba que, aplicando únicamente la pedagogía, su hijo se convertirá indefectiblemente en un genio (Unamuno 1994, 32). Hay que transmitir el interés por los asuntos filosóficos, el deseo por la filosofía, hacer ver su necesidad, antes que deslumbrar y abrumar con desorientadora información. Como decía Ortega, "el problema de la pedagogía no es educar al hombre exterior, al anthropos, sino al hombre interior, al hombre que piensa, siente y quiere" (MarTínez I988, 149).

\section{LO SENTIENTE EN LOS PROCESOS DE CAPACITACIÓN}

Si de lo que se trata es de hacer descender la filosofía del plano meramente intelectual al experiencial, las categorías que se ponen en juego no serán únicamente las intelectivas, sino que a ellas se les deben añadir las relacionadas con esa dimensión vital o biográfica. Para realizar este paso, el pensamiento 
zubiriano nos ofrece un valioso marco, ya que en él se conjugan unitariamente las categorías intelectivas con las sentientes.

En las últimas páginas de Inteligencia y razón (último volumen de su famosa trilogía) Zubiri resume estos ámbitos que comentábamos (un saber acerca de las cosas, una orientación para el mundo y un modo de vida) en uno solo: saber estar en la realidad. Y mientras que en el resto de seres vivos ese saber es algo dado por sus propias estructuras constitutivas, 'estar en la realidad' constituye para el ser humano un auténtico problema; es más: su vida y lo que haga en ella no es sino un intento más o menos afortunado de responder a esta cuestión fundamental. Sobre ese cuadro de fondo dibujado por el reino animal, confinado en diferentes ambientes por instintos fijos e innatos, y con una capacidad de aprendizaje que opera también en este marco limitado (GeHLen I993, 32-3), destaca la posición singular del ser humano en la naturaleza; una posición que si bien naturalmente es carencial, queda compensada gracias a su capacidad de modificar su entorno.

Para Zubiri lo característicamente humano es estar en la realidad con unas estructuras que no determinan nuestro comportamiento. Somos seres constitutivamente abiertos: nuestra especificidad pasa por un comportamiento inespecífico. Consecuentemente, nuestro despliegue vital es esencialmente diverso al del resto de seres vivos, un despliegue que se realiza — tal y como nos explica Gehlen - en un diálogo de ida y vuelta con la realidad, en un aprendizaje a base de prueba y error, de modo que fruto de esta confrontación con la realidad el ser humano puede ir alcanzando distintas grados en su desarrollo: "La acción es de por sí — diría yo- un movimiento cíclico complejo que se conecta a través de las cosas del mundo exterior, y la conducta se modifica según los resultados que avisan de vuelta" (Gehlen 1993, 34). Porque "el yo, la personalidad, emerge en interacción con los otros yo y con los artefactos y demás objetos de su entorno" (Popper \& ECCLEs i 980, 56).

Mientras este saber desenvolverse en la vida los animales lo aprenden en periodos cortos, el ser humano necesita años, entremezclándose lo estrictamente fisiológico con lo cultural. El 'mundo' humano ya no es únicamente el que le presentan sus instintos sino aquel en el que se sitúa socialmente, multiplicando exponencialmente las capacidades de adaptación y poniendo de manifiesto la plasticidad humana. A causa de esta inespecificidad, es fundamental en el desarrollo de cada individuo su ambiente, pues en función de él se le exigirán unas respuestas u otras, las cuales determinarán su propio crecimiento personal.

Nuestro crecimiento está íntimamente relacionado con la experiencia que podamos ir adquiriendo durante nuestras vidas, sobre todo en los ańos iniciales. Por la idiosincrasia de nuestra cultura, altamente civilizada, los ambientes en que nos movemos satisfacen con frecuencia nuestras necesidades 
básicas, dificultando el robustecimiento de nuestras estructuras fisiológicas primarias las cuales se fortalecen precisamente ante los obstáculos. Es por ello que se han ido solidificando nuestras categorías vitales, derivándolas hacia aspectos más intelectivos o conductuales, y relegando a un segundo nivel aquello que nos arraiga físicamente con la realidad. Y esto es algo que no ha escapado a la filosofía, ocupada principalmente en planteamientos cognitivos y éticos.

A la luz del pensamiento zubiriano se observa que la inteligencia es mucho más que su dimensión cognitiva, tanto como puede abarcar esa otra dimensión suya que el filósofo vasco considera: la sentiente. Sabemos que para Zubiri la facultad específicamente humana y que nos proporciona ese puesto singular en la naturaleza es la inteligencia sentiente: una inteligencia sentiente que no es únicamente una inteligencia sentiente sino también una inteligencia sentiente. Si en la tradición filosófica el sentir y el inteligir han seguido caminos paralelos, la propuesta zubiriana pasa por su consideración unitaria, no como resultado de una yuxtaposición sino como una única facultad: la 'inteligencia sentiente' o el 'sentir intelectivo' (Zubiri I926).

Su consideración sentiente de la inteligencia va mucho más allá de lo específicamente sensorial, de lo relacionado estrictamente con lo que nuestros sentidos fisiológicos pueden aprehender. Efectivamente, los sentidos nos permiten aprehender distintas 'parcelas' de la realidad, pero además cada uno de ellos nos permite aprehender la realidad de diverso modo: nos ofrecen no solo distintos ámbitos de inteligibilidad sino también distintos modos de intelección, de modo que considerados en su conjunto posibilitan una aprehensión de la realidad mucho más rica, así como un ejercicio de la inteligencia no únicamente lógico-científico sino también estético o poiético, muy lejos de la irracionalidad que tradicionalmente se le ha atribuido. El sentir adquiere una relevancia fundamental en tanto que nos posibilita nuevas modalidades de encuentro con lo real que no tienen por qué estar enfrentadas con lo intelectivo, todo lo contrario.

Ello evidencia a su vez la importante carencia de una personalidad humana que no considere esta dimensión sentiente. Es preciso, pues, recuperar una pedagogía del sentir (NIÑo 2009), porque mediante ella podemos establecer un diálogo más completo con la realidad, diálogo que necesitamos establecer inevitablemente para nuestro propio crecimiento. Nuestra personalidad se construye en diálogo con la realidad, fruto del cual inexorablemente se configura nuestra sustantividad humana.

Esta configuración-construcción no es mera maduración, entendida esta como aquel proceso según el cual se desarrollan unas virtualidades que ya se encontraban germinalmente al inicio y solo precisa del despliegue vital para que broten. Este es un proceso que puede darse en el desarrollo biológico o 
fisiológico del ser humano, pero no abarca el desarrollo de toda la persona: sin maduración biológica no habría persona, pero el desarrollo de la persona no puede ser reducido a su maduración biológica. El desarrollo personal lo articula Zubiri alrededor del proceso de capacitación. Capacitación... ¿de qué? Todos los seres humanos compartimos unas mismas potencias y facultades, pero no todos las ejercemos por igual. Si bien el ser humano nace con unas facultades que le son dadas, él puede desarrollarlas o minimizarlas en función de su biografía y de su historia. Capacitación... ¿para qué? Pues para poseer mayores posibilidades a la hora de hacer nuestras vidas. Según cada individuo esté más o menos capacitado podrá descubrir más o menos posibilidades en su diálogo con la realidad.

Gracias a este proceso alcanzamos un determinado grado en el ejercicio de nuestras facultades, según dos dimensiones: una dimensión operativa (disposiciones) y una dimensión constitutiva (estrictamente capacidades). Las capacidades tienen que ver no con nuestro grado en el ejercicio operativo de una facultad, sino con la misma facultad en tanto que principio de posibilitación. El ser humano descubre en las cosas reales (y en las personas) posibilidades que van más allá de sus meras propiedades físico-químicas, con las que hace su vida; cuando se encuentra con ellas, puede utilizarlas como meras cosas pero también puede utilizarlas según posibilidades nuevas que surgen de la relación que establece con ellas durante su despliegue vital. No se trata únicamente de utilizar las cosas para hacer nuestras vidas, sino de crear posibilidades de interacción con ellas que permitan desarrollarnos como personas, que es totalmente distinto.

Cada ser humano va definiendo por sí mismo la dirección por la que va a discurrir su vida, en el marco de su contexto histórico; desde su biografía irá definiendo la senda por la que caminará y desde la cual desarrollará sus propias capacidades. Algo de ello le será dado (tradición, educación) pero en lo restante dependerá de sí mismo. Las posibilidades que vaya descubriendo influirán en el desempeńo de su propia vida, construyendo así de forma creativa su propia personalidad: cada uno se irá haciendo a sí mismo 'a su manera'. Consecuencia de ello, podrá actuar haciéndose cargo en mayor o en menor medida de la realidad en la que está instalado, y así hacer su vida; tarea que, lejos de ser opcional, es consecuencia intrínseca de su propio modo de ser.

De lo que se trata no es tanto de aprehender posibilidades, como del hecho mismo de la posibilitación en tanto que rasgo de nuestra sustantividad. No se trata de que cada uno posea un mayor o menor espectro de posibilidades, sino de nuestra aptitud para poder descubrirlas de modo creativo en orden a la configuración de nuestra personalidad: es a esto a lo que se refiere Zubiri cuando habla de capacitación. Con lo que nos es dado, no está todo dicho. Es más, ni siquiera está dicho lo más importante: lo más importante es cómo hacemos 
nuestras esas posibilidades que nos son dadas, cómo las aplicamos a nuestras vidas (a la vez que adivinamos otras nuevas), cómo las podemos integrar $\mathrm{crea}$ tivamente en nuestras vidas. En Zubiri estos procesos poseen una circularidad dialógica: partimos de unas ciertas capacidades que nos van a permitir la aprehensión de determinadas posibilidades; y a la vez, aquellas posibilidades por las que optemos van a repercutir en la configuración de nuestra sustantividad (NIÑo 2009), favoreciendo o no la aprehensión de nuevas y más ricas posibilidades. Es un proceso en el que una parte nos es dada pero la otra no: somos así autores de nuestra propia vida.

\section{LA PEDAGOGÍA DE LA ADMIRACIÓN}

A la luz de lo expuesto, cabe concluir que la educación no debe recaer tanto sobre las posibilidades externas como sobre las internas (MARQuínEZ ARGOTE I989), pues se capacita a los educandos a crear posibilidades nuevas que puedan revertir en ellos favorablemente, tras un discernimiento libre en el seno de una realidad no únicamente inteligida sino también sentida. Una persona no capacitada debidamente es una persona limitada existencialmente. Es por ello que cualquier vida no deja de ser un proceso de capacitación (o de des-capacitación), y por ende un proceso mediante el cual adquirimos (o no) libertad creativa de vida. Por ello hay que enseñar capacidad, hay que enseñar inteligencia (Niño I983) pero no de modo eminentemente cognitivo sino también primariamente sentiente, experiencial, lo que nos permitirá considerar ese aspecto físico de la realidad fruto de ese 'bien percibir' y de ese 'bien aprehender'.

El docente de filosofía se erige así en alguien que ha sufrido una transformación personal y trata de transmitirla, ofreciendo al alumno no solo un mundo nuevo de posibilidades (filosóficas, vitales) sino también una actitud nueva ante la vida y ante sí mismo. Lejos de una vida academicista, la vida filosófica es una vida vivida en primera persona, para la cual el problema radical de su existencia se erige en guía de trabajo y de reflexión, del que deriva cualquier otro problema. La filosofía se distancia así de la mera erudición para arrimarse inexorablemente a lo vocacional y vivencial.

Este proceso de capacitación es un proceso complejo en el que intervienen tanto elementos cognitivos y volitivos como afectivos. Alfonso López Quintás propone una pedagogía de la admiración (López QuiNTÁs 2003), cuya fecundidad filosófica le dota de grandes posibilidades vitales. ${ }^{2}$ El núcleo básico

\footnotetext{
${ }^{2}$ En su página web (http://www.fundacionlopezquintas.org/) se puede encontrar copiosa información, tanto de sus publicaciones como de su amplia experiencia académica.
} 
gira en torno a ese diálogo (ya comentado) entre nuestros deseos y necesidades y las características específicas de las realidades que vamos descubriendo y con las que vamos haciendo nuestras vidas, pues nuestro despliegue no se realiza al margen de las cosas sino con ellas. De lo que se trata es de aprender a ajustar creativamente nuestras necesidades y deseos a las exigencias intrínsecas de la realidad y de nuestra realidad; un aprendizaje que no es a base de recetarios ni de normas, sino de una invitación a que cada uno lo vaya descubriendo por sí mismo, salvaguardando así en todo momento la libertad e iniciativa personales.

Ante la realidad de las cosas bien se puede adoptar una actitud de apertura y de colaboración creando relaciones de encuentro, bien una actitud de dominio y manipulación creando procesos de distanciamiento que nos dejan a la intemperie sin poder vivir la vida con un mínimo de seguridad. En opinión de López Quintás los docentes hemos de procurar transmitir la diferencia que existe entre la experiencia de 'vértigo o fascinación' que nos encamina hacia una mera 'libertad de maniobra', de la experiencia de 'éxtasis' que nos dirige hacia una auténtica 'libertad creativa'. Se trata de 'hacer nuestra' la realidad mediante las posibilidades que vamos descubriendo en ella y que revierten en nosotros positivamente, y ello pasa por vibrar con aquellas realidades que vamos descubriendo. Si no se da esa resonancia en nuestro interior no nos haremos cargo de la 'grandeza que albergan' ni las valoraremos en su verdad y, lo que quizá sea más importante, no lograremos hacer del saber un principio de excelencia personal. Todo ello pasa por saber despertar la capacidad para la admiración, paso previo para poder ejercer nuestra libertad del modo más liberador: creativamente. No es casualidad que una de sus obras más importantes se denomine Estética de la creatividad. 


\section{BIBLIOGRAFÍA}

Argullol, R. \& Djermanovic, T. 20i6, "La filosofía, arte del pensar", Razón y fe: 107-119.

Gehlen, A. I993, Antropología filosófica, Barcelona: Paidós.

López Quintás, A. 1998, Estética de la creatividad, Madrid: Rialp.

López Quintás, A. 2003, Descubrir la grandeza de la vida, Estella: Verbo Divino.

López Quintás, A. 2003, “La filosofía y su fecundidad pedagógica”, Revista Estudios: 29-253.

López Quintás, A. 2004, Liderazgo creativo, Oviedo: Nobel.

López Quintás, A. 2006, Biblioteca del educador, Buenos Aires: Puerto de Palos.

López Quintás, A. 2006, Vértigo y éxtasis, Madrid: Rialp.

López Quintás, A. (s.f.). "La pedagogía de la admiración y su fecundidad educativa”. Recuperado el 3 de marzo de 2015, de http://www.foro.santyago.org/ recursos/58-admiracion.pdf

Marquínez Argote, G. I989, "La educación como proceso de posibilitación y de capacitación en Xavier Zubiri", Cuadernos de Filosofía Latinoamericana: 75-83.

Martínez, J. A. I988, "Xavier Zubiri y su esbozo de educación intelectual”, Análisis: 137-49.

Niño, F. I983, "Inteligencia y educación en el pensamiento de Xavier Zubiri", Cuadernos de Filosofía Latinoamericana: 5-14.

NiÑo, F. 2009, "Una educación para saber estar en la realidad”, en G. MARQuíneZ, \& F. Niño, Introducción a la filosofía de Xavier Zubiri, Bogotá, D.C.: El búho, $129-44$.

Popper, K. \& Eccles, J. I980, El yo y su cerebro, Barcelona: Labor.

Ruiz López, L. E. I988, "Hacia una didáctica de la filosofía con base en Zubiri", Análisis: 287-304.

Unamuno, M. I994, Amor y pedagogía, Madrid: Club Internacional del Libro.

Zubiri, X. I926, "Filosofía del ejemplo", Revista de Pedagogía: 289-95.

ZubIRI, X. I983, Inteligencia y razón, Madrid: Alianza Editorial \& Fundación Xavier Zubiri.

Zubiri, X. I986, Sobre el hombre, Madrid: Alianza Editorial \& Fundación Xavier Zubiri.

Zubiri, X. 2006, Tres dimensiones del ser humano: individual, social, histórica. Madrid: Alianza Editorial \& Fundación Xavier Zubiri.

Zubiri, X. 2007, Naturaleza, historia, Dios. Madrid: Alianza Editorial \& Fundación Xavier Zubiri. 
